\title{
Correction to: A computational approach for the experimental study of EU case law: analysis and implementation
}

\author{
Nicola Lettieri ${ }^{1}(1) \cdot$ Antonio Altamura $^{2} \cdot$ Armando Faggiano $^{2} \cdot$ Delfina Malandrino $^{2}$
}

Published online: 23 November 2017

(c) Springer-Verlag GmbH Austria 2017

\section{Correction to: Soc. Netw. Anal. Min. (2016) 6:56 https://doi.org/10.1007/s13278-016-0365-6}

The author would like to correct the errors in the publication of the original article. The corrected details are given below for your reading.

In Abstract section, fourth sentence should read as:

The attention is focused on EuCaseNet, an online toolkit allowing legal scholars to apply NA and visual analytics techniques to the entire corpus of EU case law.

In Introduction section, first sentence should read as:

In the last few years, network science, the "study of network representations of physical, biological, and social phenomena," has gradually spread in extremely heterogeneous research areas ranging from Physics to Biology and Genetic, from Mathematics and Computer Science to Sociology and Economics.

In Background section, second sentence should read as:

Thanks to its high level of abstraction and to the ability to support the understanding of structural and functional features of real networks, NA is spreading in the legal domain through both scientific and commercial applications exploited for purposes that span from visual analytics to criminal network analysis.

The original article can be found online at https://doi. org/10.1007/s13278-016-0365-6.

Nicola Lettieri

n.lettieri@isfol.it

Antonio Altamura

antonio.altamura7@gmail.com

Armando Faggiano

armando.faggiano@gmail.com

Delfina Malandrino

dmalandrino@unisa.it

ISFOL, Rome, Italy

2 Computer Science Department, University of Salerno, Salerno, Italy
In 2.1 Visualize, analyze and retrieve legal information section, second sentence of the third paragraph should read as:

Lawiz (Lettieri et al. 2014) is a recent experimental Web application designed for the visualization, the exploration and the analysis of documents coming from heterogeneous sources of the Italian law (legislation, parliamentary works, Supreme Court case law, Constitutional case law, legal doctrine).

In Section 3.1 Analysis of the features of the ECJ judgments corpus, third sentence of the first paragraph should read as:

In more details, this set of features encompasses the following functionalities:

In Section 3.1 Analysis of the features of the ECJ judgments corpus, first bullet point should read as:

Analyze the EU case law system represented as a network. This feature allows the exploration of "network properties" of the ECJ case law corpus (properties deriving from the application of NA measures like Modularity or Betweenness) so that to discover legal properties of single judgments or group of them. The attention is focused on issues like the presence of clusters (interpretable, for instance, in semantic terms, as the sign of the influence of one judgment on a group of pronouncements).

In Section 3.2 Analyze the features of some of the most relevant ECJ judgments, second sentence of the first paragraph should read as:

The goal is twofold: visually analyze the properties of what can be considered a gold standard; implement a sort of supervised "features extraction mechanism" aiming to discover the computational landmarks of relevant precedents (e.g., by stating that relevant judgments are always characterized by high Page Rank values).

In Section 3.2 Analyze the features of some of the most relevant ECJ judgments, first sentence of the second bullet point should read as: 
Semantically, compare the topics of the gold standard with ECJ corpus' ones.

In Section 4.1.1 Network analysis and visualization of a citation network, first sentence of the section "Graph generation, visualization and analysis" should read as:

This feature has been implemented taking into account both the whole corpus of ECJ, shown in Fig. 2, and the gold standard citation network dataset, shown in Fig. 3.

In Section 4.1.2 Quantitative analysis and visualization of judgments' metadata, last sentence of the first paragraph should read as:

To achieve that, we implemented the following features:

In Section 5.1 Properties of the EU case law corpus, third sentence of the first paragraph should read as:

As pointed out in a well-established literature in this field (see, among the others, Fowler et al. 2007; Koniaris et al. 2015) the mentioned analyses can offer a good starting point for more domain-specific research questions: studies measuring legal authority, identifying authoritative precedent, evaluating the relevance of court decisions or even predicting the cases that will receive more citations in the future.

In Section 5.1 Properties of the EU case law corpus, first sentence of the fifth paragraph should read as:

As shown in Fig. 12, the approximate straight-line form of the distribution function allows us to infer that, in all the examined subgraphs, the Legislation Network follows a power-law distribution both in the in-degree and in the out-degree distribution.

In Section 5.2 Analyzing a gold standard, first paragraph should read as:

After the set of analyses described in the previous section, we decided to start facing the problem of the determination of the relevance of judgments in the specific context of EU case law. Being aware of the "multi-dimensionality" of the concept of relevance (and of the fact that its different profiles partly overlap and are often indicated by similar clues), we decided to focus on a profile of relevance that is well-identified in a recent book edited by two EU law scholars listing the "classics of the EU law" (Maduro and Azoulai 2010). The concept is summed up as follows: "The 'classic' judgments in the jurisprudence of a Court are those that survive the passing of time but also those which, even when their concrete legal answer has become obsolete or has even been overturned, have lived on through their multiple effects in other areas of law. They are judgments of systemic impact, embodying a broader normative lessons about the legal order in which that Court operates. To identify them we must look not only at the particular judgment itself but also at how it has been interpreted and developed by the Court in other cases. We also need to look at the attention paid to it by lawyers, legal scholars and other social actors. The law is also a function of how the judicial decisions are interpreted and challenged by then broader legal and social communities in which a court operates."

In Section 5.2 Analyzing a gold standard, fourth sentence of the fourth paragraph should read as:

This kind of problem is frequent when citation analysis is the only method used for identifying the relevance over long run, since it allows to detect only the explicit links (i.e., an express reference), without any consideration for those just implicitly contained in a document (e.g., the case reinterpreted as general principles of law within an argumentative path).

In Section 6 Conclusion, second paragraph should read as:

Our work, it is clear, is just at the beginning: there is a huge series of scientific and technical challenges involving different expertise from computer science and linguistics to be faced if we want to make this kind of tools and approaches capable of giving concrete answers to real-world problems. The active contribution of legal experts, obviously, will be essential if we want to understand how NA, VA and, more in general, computational approaches can shed new light on our understanding of legal phenomena.

In Section 6 Conclusion, third paragraph should read as:

Moving a bit more on to the substance of the research, we can state that, even if very preliminary, our results show that Network analysis measures offer poor performances when applied on a citation-based network. Further investigation will be therefore needed to understand how to better exploit NA approach in the analysis of issues like case law relevance.

In Section 6 Conclusion, first sentence of the sixth paragraph should read as:

Another crucial essential aspect will be the development of a more and more refined operational/computational definition of legal research questions (starting from the concept of relevance) to be addressed by means of visualization and NA techniques identifying also the data sources more suitable to this aim.

In References section, the following reference should be included:

Maduro M, Azoulai L (eds) (2010) The past and future of EU law: the classics of EU law revisited on the 50th anniversary of the Rome Treaty. Hart Publishing. 